\title{
Research on the injection of new gases in Underground methane Storage Systems such as deep aquifers.
}

\author{
P. HADDAD ${ }^{1 *}$, A. RANCHOU-PEYRUSE ${ }^{2}$, F. \\ CASTERAN $^{1}$,M. GUIGNARD ${ }^{2}$, P. CHIQUET ${ }^{3}, \mathrm{G}$. \\ CAUMETTE $^{3}$, D. DEQUIDT ${ }^{4}$, P. CEZAC ${ }^{1}$.
}

${ }^{1}$ University of Pau and Pays de l'Adour, E2S UPPA, LaTEP, Pau, Fr (* perla.haddad@univ-pau.fr)

${ }^{2}$ University of Pau and Pays de l'Adour, E2S UPPA-CNRS, IPREM, Pau, Fr

${ }^{3}$ TEREGA, 40 avenue de l'Europe-CS20522- $64000 \mathrm{Pau}, \mathrm{Fr}$

${ }^{4}$ STORENGY, Geosciences Department, 12 rue Raoul

Nordling CS70001, 92274 BOIS-COLOMBES, Fr

Biomethane Storage in UGS

Oxygen $\left(\mathrm{O}_{2}\right)$ is among the biomethane impurities that has impacts during the storage of biomethane in the underground storage systems (UGS), such as aquifers. Interactions of oxygen in the anaerobic aquifers can modify its biogeochemical conditions [1],[2]. Due to the lack of information, an experimental program is conducted with a view to reproduce deep aquifers in situ conditions and to observe the effect of $\mathrm{O}_{2}$ injection. Results obtained will help define the quality standards required for biomethane storage.

\section{Technology Description}

Our simulated aquifer consists of a batch reactor. Three phases are considered: an anaerobic groundwater sampled (with autochthonous microorganisms) from the aquifer, rock samples from the reservoir; and a gas phase simulating the natural gas with $99 \%$ methane and $1 \% \mathrm{CO}_{2}$, at a pressure of 60 bars. After reaching an equilibrium, $\mathrm{O}_{2}$ at $1 \%$ was injected. Thermodynamic studies and microbial analysis are conducted.

\section{Discussion of Preliminary Results}

During the first months of incubation in in situ conditions, the disappearance of sulfate was measured and undoubtedly consumed by the sulfate-reducers, key players of the microbial communities in deep subsurface. After adding $\mathrm{O}_{2}$, sulfate reduction stopped, sulfate and $\mathrm{H}_{2}$ appeared.Those results with microbiological, mineral and tomography analysis prove that the presence of oxygen in UGS has multiple effects on the biogeochemical equilibrium of the system. One of the effects can be a change in the aquifers microcosm, influencing the system's established equilibrium.

[1] H. Morgan et al. (2017), Energy Procedia 114. [2]S. Berlendis et al. (2010), Environmental Science \& Technology 44, no 9. 